\title{
Rare beneficial mutations cannot halt Muller's ratchet in spatial populations
}

\author{
Su-Chan Park ${ }^{1}$, Philipp Klatt ${ }^{2}$ and Joachim Krug ${ }^{2}$ \\ 1 Department of Physics, The Catholic University of Korea - 43 Jibong-ro, Bucheon 14662, Republic of Korea \\ 2 Institut für Biologische Physik, Universität zu Köln - Zülpicher Str. 77, 50937 Köln, Germany
}

PACS 87.23.Kg - Dynamics of evolution

PACS 64.60.Ht - Dynamic critical phenomena

PACS 05.70.Ln - Nonequilibrium and irreversible thermodynamics

\begin{abstract}
Muller's ratchet describes the irreversible accumulation of deleterious mutations in asexual populations. In well-mixed populations the speed of fitness decline is exponentially small in the population size, and any positive rate of beneficial mutations is sufficient to reverse the ratchet in large populations. The behavior is fundamentally different in populations with spatial structure, because the speed of the ratchet remains nonzero in the infinite size limit when the deleterious mutation rate exceeds a critical value. Based on the relation between the spatial ratchet and directed percolation, we develop a scaling theory incorporating both deleterious and beneficial mutations. The theory is verified by extensive simulations in one and two dimensions.
\end{abstract}

Introduction . - The evolution of asexual populations is driven by novel mutations as the sole source of genetic diversity. Although the vast majority of mutations is expected to decrease fitness, these deleterious mutations often play only a minor role because they are efficiently purged by natural selection. However, in small populations deleterious mutations may spread and fix due to stochastic drift. When all mutations are deleterious this leads to an irreversible decline of the fitness of the population that is known as Muller's ratchet.

The ratchet mechanism was first described verbally by Hermann Muller in the context of identifying possible evolutionary advantages of genetic recombination [1. Recombination counteracts the accumulation of deleterious mutations, because the number of mutations of an individual (its mutational load) can be reduced by recombining with another individual that carries mutations at different genetic loci. The standard mathematical formulation of Muller's ratchet considers an asexual population that is well-mixed, in the sense that competition between individuals is implemented only through the constraint of constant population size $N$. Deleterious mutations occur at rate $U_{d}$ per individual and generation, and each mutation decreases the fitness of the individual by a constant factor that we denote by $e^{-s}$ with $s>0$ [2, 3. Despite the simplicity of the model, the computation of the speed of fitness decline as a function of $N, U_{d}$ and $s$ is a hard problem that has attracted the attention of population geneticists for more than 40 years 4 -11.

A key parameter governing the behavior of the ratchet is the deterministic expectation of the number of individuals that carry the smallest mutational load, which is given by 3

$$
n_{0}=N e^{-U_{d} / s}
$$

A click of the ratchet occurs when the least loaded class goes extinct and the minimal number of deleterious mutations carried by any individual increases by one. When $n_{0} \gg 1$ this event is rare and the fitness declines slowly, whereas for $n_{0} \sim 1$ the decline is rapid and continuous. Importantly, for sufficiently large populations the speed of the ratchet becomes immeasurably small, irrespective of the values of $U_{d}$ and $s$. Correspondingly, in large populations any positive rate $U_{b}>0$ of beneficial mutations is sufficient to halt and reverse the ratchet such that the fitness of the population increases [7, 12.

Here we show that the scenario is fundamentally different in spatial populations, where the competition between individuals is limited to their local neighborhood. Spatial models of adaptive evolution driven by beneficial mutations have been developed in various contexts, and a number of characteristic features have been identified that differ from the well-mixed setting 13 21. In particular, provided the density of individuals is bounded, the speed 
of adaptation remains finite when the habitat size tends to infinity [17. This is in contrast to well-mixed populations, where the speed of fitness increase diverges logarithmically with the population size [7, 12,22.

The effect of spatial structure is even more pronounced for the accumulation of deleterious mutations. It was shown in 23. that the spatial Muller's ratchet in an infinite habitat displays a sharp phase transition at a critical value $U_{d}^{c}$ of the deleterious mutation rate such that the fitness declines at a finite rate for $U_{d}>U_{d}^{c}$ and remains constant for $U_{d}<U_{d}^{c}$. In the following these two dynamical states will be referred to as the moving ratchet (MR) regime and the halting ratchet (HR) regime, respectively.

The existence of a phase transition in the spatial ratchet, together with the observation that the speed of adaptation is bounded and vanishes when the beneficial mutation rate tends to zero, suggests that a small amount of beneficial mutations will not be able to halt or reverse the ratchet when $U_{d}>U_{d}^{c}$. The purpose of this Letter is to verify and corroborate this conjecture using extensive simulations in one and two-dimensional habitats. Based on these simulations and the known relation of the spatial Muller's ratchet problem to directed percolation and nonequilibrium wetting 23, we develop a scaling theory for the (positive or negative) speed of fitness change as a function of the parameters $U_{d}, U_{b}$ and $s$. In the next section we introduce the spatial evolution model and outline its relation to other problems in nonequilibrium statistical physics. We then present our scaling theory and the numerical results, and conclude the paper with a summary and a discussion of some biological implications.

Model. - We first define the spatial model in a general setting and later specify the rules that are suitable for our purpose. We consider a system with $N$ sites, each of which is indexed by an integer $x=1,2, \ldots, N$. At each site, a single individual is accommodated and the individual is characterized by its fitness $w_{x}$. By $\mathcal{N}_{x}$ we denote the set of indices of nearest neighbors of site $x$. For later purposes the union of $\mathcal{N}_{x}$ and $\{x\}$ is denoted by $\mathcal{A}_{x}$. Our main interest is in the behavior of a population in the infinite $N$ limit.

We consider a nonoverlapping-generation model with parallel update in the spirit of Wright and Fisher (WF) [22, 24, 25]. If the fitness at site $x$ is $w_{x}$ in generation $t$, the fitness distribution in the next generation $t+1$ is determined in two steps.

Selection step: Fitness at site $x$ is replaced by fitness at site $y$, where $y$ is chosen among $\mathcal{A}_{x}$ according to the probability

$$
S_{x, y}=w_{y}\left(\sum_{z \in \mathcal{A}_{x}} w_{z}\right)^{-1}
$$

Needless to say, $y$ can be $x$ itself. The fitness values at all sites are updated simultaneously.
Mutation step: After the selection step, the fitness at every site can be modified by mutation. We denote the mutation probability (density) by $\mu\left(w^{\prime} \mid w\right)$ which means that fitness becomes $w^{\prime}$ if $w$ is the fitness after the selection step.

In this Letter, we limit ourselves to the case that fitness takes the form $w_{x}=e^{s h_{x}}$, where $s$ is a nonnegative real number and $h_{x}$ is an integer, and mutation can change $h_{x}$ by an integer value $m$ with probability $U(m)$. That is, $\mu\left(w^{\prime} \mid w\right)=\sum_{m} U(m) \delta\left(w^{\prime}-w e^{s m}\right)$, where $m$ runs over all integers. In the following, $s$ will be called the selection coefficient and $h_{x}$ will be referred to as the number of mutations, where deleterious mutations are counted negatively. For convenience (and anticipating the connection of this model to surface growth models), we will also call $h_{x}$ the height (at $x$ ). This mutation scheme is multiplicative in terms of the $w_{x}$ and additive in terms of the $h_{x}$, which implies that epistatic interactions between mutations are excluded 26].

To be specific, we define $U(m)$ as the convolution of two probabilities $\mu_{b}(m)$ and $\mu_{d}(n)$ with

$$
\begin{aligned}
\mu_{d}(n) & =\frac{U_{d}^{-n}}{(-n) !} e^{-U_{d}}, \\
\mu_{b}(m) & =\left(1-U_{b}\right) \delta_{m, 0}+U_{b} \delta_{m, 1},
\end{aligned}
$$

where $1 / k$ ! with a negative integer $k$ is interpreted as 0 , and $U_{d}$ and $U_{b}$ denote the probabilities of deleterious and beneficial mutations. The Poisson distribution for deleterious mutations is the typical choice in studies of Muller's ratchet [3], but other forms of $\mu_{d}$ will not change our conclusions as long as $U_{d} \ll 1$.

Speed of fitness change. We are mainly interested in the speed of fitness change which is defined as

$$
v(t)=\frac{1}{N} \sum_{x}\left\langle\frac{d h_{x}}{d t}\right\rangle, V_{h}=\lim _{t \rightarrow \infty} v(t), V_{A}=s V_{h} .
$$

Here the time derivative of $h_{x}$ should be understood as $h_{x}(t+1)-h_{x}(t)$ and $\langle\ldots\rangle$ stands for an average over all realizations. Whereas trivially $V_{A}=0$ if $s=0, V_{h}$ can be nonzero even for $s=0$. We will therefore focus on the more informative quantity $V_{h}$ in the following. For $V_{h}>0$ the population adapts whereas for $V_{h}<0$ it is subject to fitness decline.

For the general class of models defined above it can be shown that the speed is related to the steady state distribution of the height configurations as [27]

$$
v(t)=M+\left\langle\sum_{y \in \mathcal{N}_{x}}\left(h_{y}-h_{x}\right) S_{x, y}\right\rangle,
$$

where $M$ is the average change of height by mutations per generation per capita, that is, $M \equiv \sum_{m} m U(m)$, and the average on the right hand side is taken at generation $t$. For the mutation scheme in Eq. (3), $M=U_{b}-U_{d}$. 
This equation has a simple interpretation. The contribution to the speed is twofold, by mutation and selection. Since mutation and selection are operating separately, the contributions just add up. Obviously, mutation changes the height by $M$ on average. Selection can change the height at site $x$ only if a neighbor is chosen as a parent. For a given configuration, the increase is $h_{y}-h_{x}$, which happens with probability $S_{x, y}$. Hence we obtain Eq. (5).

The spatial structure is determined by $\mathcal{N}_{x}$. For the one dimensional system we take $\mathcal{N}_{x}=\{x+1\}$ with periodic boundary conditions as in Ref. 23 . For two and higher dimensional systems, we distribute the sites on a hypercubic lattice with size $N=L^{D}$ and choose $\mathcal{N}_{x}$ as the conventional nearest-neighbor neighborhood with periodic boundary conditions. If $\mathcal{A}_{x}$ is the set of all indices for any $x$, the model becomes the well-mixed WF model whose infinite population-size limit has an exact solution [22,28]. In this case Eq. (5) is identical to the relation first obtained by Guess [29] (see also Ref. [30] for the case with recombination). In all the simulations reported below we will use (5) to determine the speed, but fitting the mean height by a linear function gives consistent results.

Relation to other models. The model without beneficial mutations, that is $U_{b}=0$, is related to other wellstudied models of statistical physics 31. Consider first the case $U_{d}=0$. If $s$ is allowed to take also negative values and $h_{x}$ is restricted to two consecutive heights (say, -1 and 0 ) as initial condition, the model becomes equivalent to a biased voter model or compact directed percolation (CDP) 18, 32 34. For $s>0(s<0)$ the system converges to the uniform state $h_{x} \equiv 0\left(h_{x} \equiv-1\right)$, and the CDP critical point is located at $s=s_{0}=0$. If $U_{d}$ and $s$ are both positive and we choose the initial condition as $h_{x}=0$ for all $x$, the model becomes a growth model with a nonequilibrium wetting transition of the kind first studied in 35, 36. The critical behavior of such models can be described by a multilayer extension of directed percolation (DP) known as unidirectionally coupled directed percolation (UCDP) [37, 38]. In particular, the depinning of the surface from the initial level $h_{x}=0$ that occurs above a critical value $U_{d}^{c}$ is driven by the extinction of a DP process defined in the $h_{x}=0$ layer.

In the present context this implies that the deleterious mutation rate $U_{d}$ mediates a crossover from CDP to DP [19. According to scaling theory, the critical point is expected to be shifted by an amount $s_{c}-s_{0} \sim U_{d}^{1 / \phi}$ for small $U_{d}$, where $\phi$ is the crossover exponent 39.40. Since $\phi=2 / D$ for $D<2, \phi=1$ for $D \geq 2$, and $s_{0}=0$, we get

$$
U_{d}^{c} \sim \begin{cases}s^{2} & D=1 \\ s & D \geq 2\end{cases}
$$

with logarithmic corrections for $D=2$ 39. The scaling of $U_{d}^{c}$ with $s$ was previously derived and verified numerically in [18, 23 for $D=1$ and in 19 for $D=2$. The fact that $U_{d}^{c} \sim s$ for $D>2$ is consistent with the scaling of $s$ and

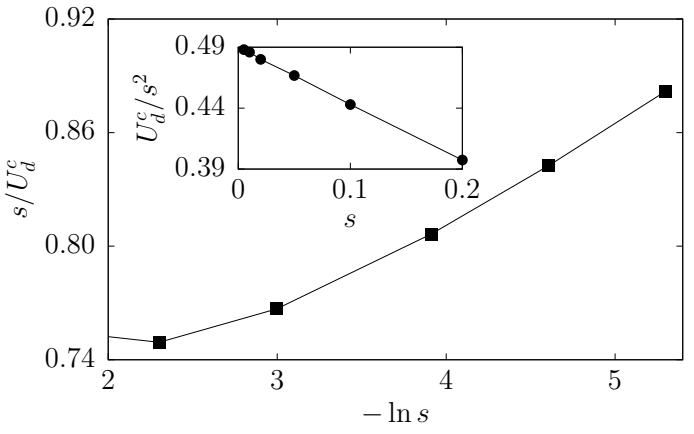

Fig. 1: Plot of $s / U_{d}^{c}$ vs $-\ln s$ for the two-dimensional system. Inset: Plot of $U_{d}^{c} / s^{2}$ vs $s$ for the one dimensional system.

$U_{d}$ in the well-mixed population, see Eq. (10). However, in that case there is no phase transition at any finite value of $U_{d}$.

Results. - In this section we present and interpret our simulation results. We first determined the critical point $U_{d}^{c}$ for various values of the selection coefficient $s$ by exploiting the fact that the density $\rho_{0}(t)$ of sites with $h_{x}=0$ decays as $t^{-\delta}$ at the critical point, where $\delta$ is the critical decay exponent of DP. The numerical values of $\delta$ are $\approx 0.159464$ [41] and 0.4510 [42 for $D=1$ and $D=2$, respectively. We located the critical point by analyzing how $\rho_{0}(t) t^{\delta}$ behaves with time. It should veer up (down) if the system is in the $\mathrm{HR}(\mathrm{MR})$ regime and saturate to a constant if the system is at the critical point.

Using these estimates we verified the validity of the crossover scaling (6). To confirm the logarithmic correction in two dimensions we plot $s / U_{d}^{c}$ as a function of $-\ln s$ in Fig. 1, In the inset of Fig. 1, we also plot $U_{d}^{c} / s^{2}$ as a function of $s$ for the one-dimensional case. As $s$ decreases, $U_{d}^{c} / s^{2}$ approaches a constant with a finite slope, which indicates that the leading term of corrections to scaling is $\sim s$. That is, $U_{d}^{c} \sim s^{2}\left(c_{1}+c_{2} s\right)$ with (nonuniversal) constants $c_{1} \approx 0.49$ and $c_{2} \approx 0.46$.

Scaling theory. As was mentioned previously, the model with $U_{b}=0$ shares the (universal) critical behavior of the nonequilibrium wetting models of Refs. 35, 36. When $U_{d} \neq U_{d}^{c}$, the system has a single time scale $\left|U_{d}-U_{d}^{c}\right|^{-\nu_{\|}}$, where $\nu_{\|}$is the correlation time exponent of DP. Numerical values of $\nu_{\|}$are 1.733847 [41] and 1.287 [42] for one and two dimensions, respectively. If $U_{d}>U_{d}^{c}$, the ratchet is moving and the speed is proportional to the inverse of the characteristic time scale, because this is the time scale on which the currently least loaded type goes extinct. Accordingly, the speed in the MR regime is $V_{h} \sim\left(U_{d}-U_{d}^{c}\right)^{\nu_{\|}}[23$.

Based on these considerations we can make a scaling ansatz

$$
v(t)=\left|\Delta_{d}\right|^{\nu_{\|}} G_{0}\left(\Delta_{d} t^{1 / \nu_{\|}}\right)
$$

where $\Delta_{d} \equiv U_{d}-U_{d}^{c}$, and $G_{0}$ is a scaling function with 


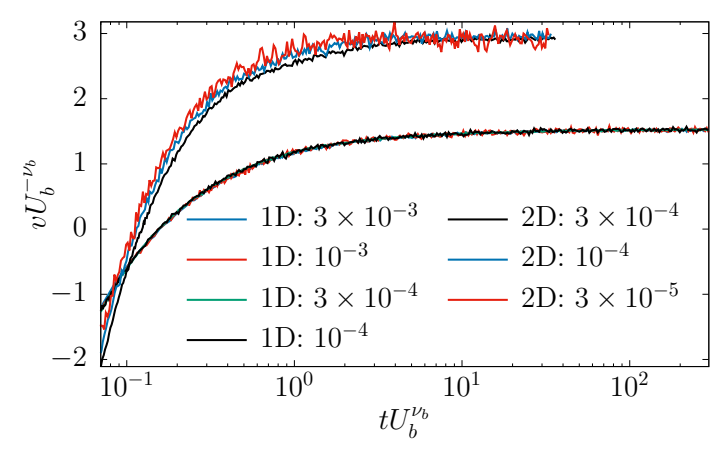

Fig. 2: Semilogarithmic plots of $v U_{b}^{-\nu_{b}}$ vs $t U_{b}^{\nu_{b}}$ at the critical point with $\nu_{b}=0.76$ and 0.81 for the one-dimensional (bottom) and two-dimensional (top) systems, respectively. The selection coefficient is $s=2.5$ and the corresponding deleterious mutation rates are $U_{d}^{c} \approx 0.304725(D=1)$ and $U_{d}^{c} \approx 1.215433$ $(D=2)$. The values for the beneficial mutation rate $U_{b}$ are displayed in the figure.

the (anticipated) asymptotic behavior

$$
G_{0}(x) \sim \begin{cases}|x|^{-\nu_{\|}}, & x \rightarrow 0, \\ (\text { negative }) \text { const }, & x \rightarrow \infty, \\ 0, & x \rightarrow-\infty\end{cases}
$$

Note that this predicts that the speed decays as $t^{-1}$ at the critical point, which corresponds to the logarithmic growth of the mean height 43 .

For nonzero $U_{b}$, the scaling ansatz needs to be extended to incorporate the effect of beneficial mutations. Assuming that $U_{b}$ affects the speed in a power-law fashion, we can write

$$
v(t)=t^{-1} F\left(\Delta_{d} t^{1 / \nu_{\|}}, t U_{b}^{\nu_{b}}\right)
$$

where $\nu_{b}$ is a new exponent and the relation between $F$ and $G_{0}$ is $F(x, 0)=G_{0}(x)|x|^{\nu_{\|}}$. At the critical point $\left(\Delta_{d}=0\right)$, we rewrite $v(t)$ as

$$
v(t)=t^{-1} F\left(0, t U_{b}^{\nu_{b}}\right)=U_{b}^{\nu_{b}} G\left(t U_{b}^{\nu_{b}}\right),
$$

where $G(x)=F(0, x) / x$. The scaling ansatz suggests that a data collapse is expected when $v U_{b}^{-\nu_{b}}$ is plotted against $t U_{b}^{\nu_{b}}$ for various $U_{b}$ 's. In Fig. 2, we indeed observe a data collapse when we set $\nu_{b}=0.76\left(\nu_{b}=0.81\right)$ for the one-dimensional (two-dimensional) system. By observing where the scaling collapse becomes worse, we conclude that $\nu_{b}=0.76 \pm 0.03$ in one dimension and $\nu_{b}=0.81 \pm 0.03$ in two dimensions, and the behavior of the speed $V_{h}$ at the critical point for nonzero $U_{b}$ is $V_{h} \sim U_{b}^{\nu_{b}}$. The seeming deviation of the collapse of the $2 \mathrm{D}$ system in comparison to the $1 \mathrm{D}$ system is due to strong corrections to scaling in the two-dimensional case.

At the critical point, $t_{b} \equiv U_{b}^{-\nu_{b}}$ is the unique characteristic time scale of the system. If the system is not at the critical point, there is another time scale $t_{c} \equiv\left|\Delta_{d}\right|^{-\nu_{\|}}$. Since the speed $V_{h}$ is inversely proportional to the characteristic time scale (as long as it is nonzero), the behavior

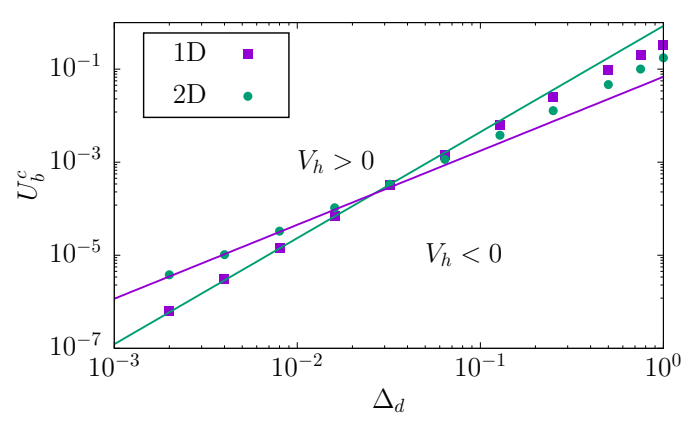

Fig. 3: Plots of $U_{b}^{c}$ vs $\Delta_{d}$ for one- (square) and two-dimensional (circle) systems for $s=2.5$. The straight lines show Eq. (12) with the numerical values for $\varphi$ mentioned in the text.

of $V_{h}$ will be determined by the smaller of the two time scales. If $t_{b} \ll t_{c}, V_{h}$ is dominated by the behavior at the critical point, whereas if $t_{b} \gg t_{c}$ it is dominated by the off-critical behavior for $U_{b}=0$. In this sense, there is a crossover from the DP type behavior to a behavior dominated by beneficial mutations at $t_{b} \simeq t_{c}$, or $U_{b}^{1 / \varphi} \simeq\left|\Delta_{d}\right|$, where $\varphi=\nu_{\|} / \nu_{b}$ is a crossover exponent. In one (two) dimension, we get $\varphi \approx 2.28$ (1.59). From these considerations, the asymptotic speed $V_{h}$ is expected to take the form

$$
V_{h}=c_{s}^{-1} U_{b}^{\nu_{b}} H\left(a_{s} \Delta_{d} U_{b}^{-1 / \varphi}\right)
$$

where $H$ is a universal function with geometric constants $c_{s}$ and $a_{s}$, which are determined by requiring $H(1)=0$ and $H(0)=1$.

The scaling ansatz (11) suggests another way of finding $\varphi$ by studying how the solution $U_{b}^{c}$ of $V_{h}\left(U_{b}\right)=0$ behaves as $\Delta_{d}$ varies. Since $U_{b}^{c}$ quantifies the beneficial mutation rate that is required to reverse the ratchet, we will refer to it as the turning point. From the scaling ansatz, we expect

$$
U_{b}^{c} \approx\left(a_{s} \Delta_{d}\right)^{\varphi} .
$$

In Fig. 3, we depict $U_{b}^{c}$ as a function of $\Delta_{d}$ for $s=2.5$. The power-law behavior for small $\Delta_{d}$ is consistent with the numerical estimate of $\varphi$ mentioned above.

In Figs. 4 and 5 , we show the scaling collapse predicted by (11) for one- and two-dimensional systems, respectively. The number of individuals is $N=2^{18}\left(2^{20}\right)$ for the onedimensional (two-dimensional) simulations. The number of independent runs for each data point is in the range $400-1000$ for the one-dimensional model and 5000 - 7000 for the two dimensional model. The initial condition is always $h_{x}=0$ for all $x$. By adjusting the geometric constants $a_{s}$ and $c_{s}$ for different $s$, all data are indeed collapsed into a single curve for various values of $s$. The curves possess two branches corresponding to the MR and HR regimes. Along the lower MR branch the speed changes sign at the turning point $U_{b}^{c}$.

We also analyzed how $a_{s}$ and $c_{s}$ behave for small $s$. As shown in the insets of Figs. 4 and 5 , we found power-law 


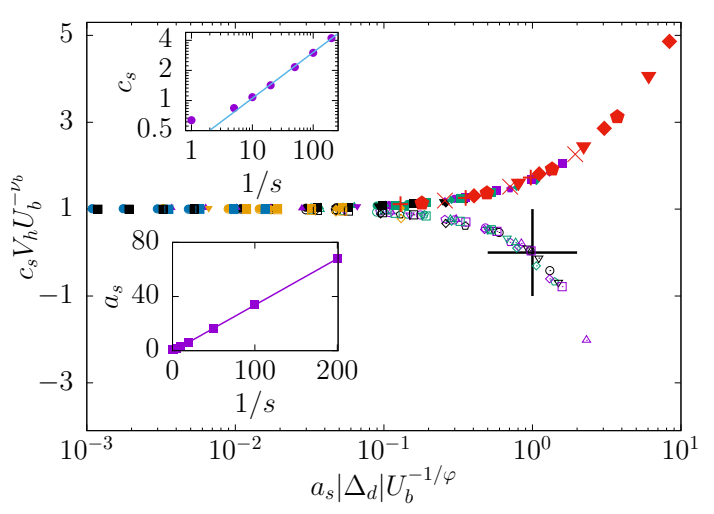

Fig. 4: Scaling collapse plot of $c_{s} V_{h} U_{b}^{-\nu_{b}}$ vs $a_{s}\left|\Delta_{d}\right| U_{b}^{-1 / \varphi}$ for the one dimensional model for $s=2.5$ (square), 1 (circle), 0.2 (triangle), 0.1 (inverted triangle), 0.05 (diamond), 0.02 (pentagon), $0.01(\times)$, and $0.005(+)$ on a semilogarithmic scale. Solid (empty) symbols are results in the HR (MR) regime. The large cross formed by a vertical and a horizontal line segment marks the position of the turning point, which is $(1,0)$ in the rescaled variables. Large symbols (in red color) indicate simulation results without deleterious mutations. Inset: (bottom) Plot of $a_{s}$ vs $1 / s$. (top) Double logarithmic plot of $c_{s}$ vs $1 / s$. The straight line is the result of a two-parameter fit $c_{s} \approx C s^{-\gamma}$ with $\gamma \approx 0.46$ and $C=0.35$.

behaviors $a_{s} \sim s^{-\alpha}$ and $c_{s} \sim s^{-\gamma}$ with $\alpha \approx 1$ (0.33) and $\gamma \approx 0.46(0.16)$ for $D=1(D=2)$. Including also the prefactor in the relation for $a_{s}$ we conclude that the turning point in the one-dimensional system is located approximately at $U_{b}^{c} \approx\left(0.34 \Delta_{d} / s\right)^{2.28}$. Due to the substantial logarithmic corrections, obtaining an accurate approximation formula for $U_{b}^{c}$ in two dimensions would require more extensive simulations.

Relation to DP exponents. The numerical estimates of $\nu_{b}$ presented above are very close to the value of $1 /(1+\eta)$, where $\eta$ is the initial slip exponent of the DP universality class, with $\eta \approx 0.313686[1 /(1+\eta) \approx 0.7612]$ in one dimension [41] and $\eta \approx 0.2307[1 /(1+\eta)=0.813]$ in two dimensions 42 . Here we present an argument in favor of this relation.

For this we need to recall the relation of the model with $U_{b}=0$ to UCDP 37,38. The connection is clearly seen by defining that site $x$ is occupied by species $A_{m}$ if $h_{x} \geq-m$ $(m \geq 0)$. Each species undergoes its own DP process. In order to maintain a well-defined height function it is assumed that a site occupied by species $m$ is also occupied by all species $l>m$. In our setting the coupling between the species is not strictly unidirectional, because the probability that $A_{m}$ increases by selection is affected by species with $l>m$. However, this feedback, which is present also in some nonequilibrium wetting models, was argued to be irrelevant in the renormalization group sense 38 .

By contrast, beneficial mutations induce a direct reverse coupling by which particles of species $m$ induce the creation of particles of species $l<m$. We start from the observation that the critical behavior of the species at the

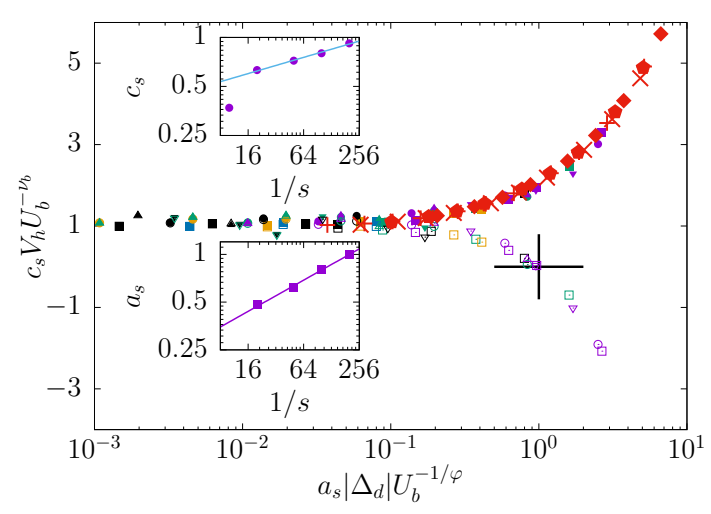

Fig. 5: Scaling collapse plot of $c_{s} V_{h} U_{b}^{-\nu_{b}}$ vs $a_{s}\left|\Delta_{d}\right| U_{b}^{-1 / \varphi}$ for the two-dimensional model on a semilogarithmic scale. We use the same symbols as in Fig. 4 for different values of $s$. Inset: Double logarithmic plots of (bottom) $a_{s}$ and (top) $c_{s}$ vs $1 / s$. The straight lines are results of power-law fittings with slopes $\alpha=0.33$ and $\gamma=0.16$ for $a_{s}$ and $c_{s}$, respectively.

lowest level is identical to DP. Let us start the process at height $h_{x}=0$ and place the system at the critical point $U_{d}^{c}$ with $0<U_{b} \ll 1$. At generation $t=1$, the density of sites with $h_{x}=1$ is then roughly $U_{b}$. In terms of the UCDP, the new species $A_{-1}$ now becomes the lowest level species which should perform the defect dynamics of DP at the critical point. It follows that the density $\rho_{-1}$ of species $A_{-1}$ at generation $t$ is $\rho_{-1}(t) \sim U_{b} t^{\eta}$. Notice that $\eta$ already incorporates the effect of deleterious mutations, which correspond to the death of $A_{-1}$ particles. Since $A_{-1}$ can change into $A_{-2}$ by another beneficial mutation which occurs with probability $U_{b}$, the expected density of species $A_{-2}$ up to generation $t$ is $\rho_{-2}(t) \sim U_{b}^{2} t^{1+\eta}$. If we define $t_{b}$ as the time when $\rho_{-2}\left(t_{b}\right)=U_{b}$, which satisfies $t_{b} \sim U_{b}^{-1 /(1+\eta)}$, species $A_{-2}$ at generation $t_{b}$ takes the place of $A_{-1}$ at generation $t=1$. Hence, we conclude that the mean height of the whole system increases by one at generation $t_{b}$, which gives $V_{h} \sim U_{b}^{1 /(1+\eta)}$ at the critical point and therefore $\nu_{b}=1 /(1+\eta)$. Since we neglected the effect of increasing $A_{-2}$ by its own dynamics as well as the dynamics of higher level species, this argument is not exact. Nevertheless our numerical estimates lend strong support to the proposed scaling relation.

Asymptotics of the scaling function. To complete the scaling theory, it remains to determine the asymptotic behaviors of the scaling function $H(x)$ in Eq. (11). This is straightforward in the $\mathrm{MR}$ regime $(x>0)$. Because $V_{h} \sim \Delta_{d}^{\nu_{\|}}$for $U_{b}=0$ and $\Delta_{d}>0$, it is expected that $H(x) \sim x^{\nu_{\|}}$for large $x$. As a consequence, $V_{h}$ becomes independent of $U_{b}$ in this regime.

The behavior for $x \rightarrow-\infty$ can be inferred from known results for the case of adaptation in the presence of purely beneficial mutations [17. Since selection should dominate when $U_{d} \ll U_{b}$, it is plausible to assume that the speed is continuous at $U_{d}=0$ for positive $s$ and $U_{b}$. This suggests that the speed for the model without deleterious muta- 


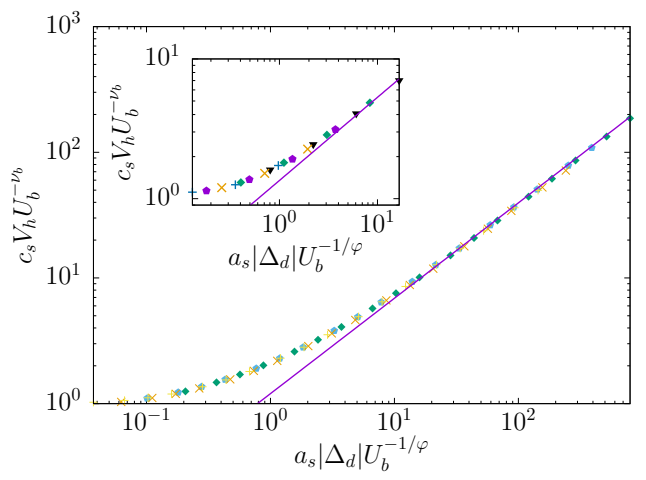

Fig. 6: Double logarithmic plot of $c_{s} V_{h} U_{b}^{-\nu_{b}}$ vs $a_{s}\left|\Delta_{d}\right| U_{b}^{-1 / \varphi}$ for the two dimensional model at $U_{d}=0\left(\Delta_{d}=-U_{d}^{c}\right)$. The slope $\chi$ of the straight line takes the value predicted by Eq. (13). The inset shows the same plot for the one dimensional case.

tions should also conform to the scaling ansatz Eq. (11), as long as $s, U_{b}$, and $U_{d}^{c}$ are small. Figures 4 and 5 include simulation results for $U_{d}=0$ (with bigger symbol size) and indeed confirm this anticipation. Assuming therefore that the case $U_{d}=0\left(\Delta_{d}=-U_{d}^{c}\right)$ is representative of the $\Delta_{d}<0$ (HR) regime, we conclude that the scaling $V_{h} \sim U_{b}^{1 /(1+D)}$ known from the model without deleterious mutations [17] should be recovered when $\left|\Delta_{d}\right| U_{b}^{-1 / \varphi}$ is large. Thus, we expect for large $-x$ that $H(x) \sim|x|^{\chi}$ with

$$
\chi=\nu_{\|}\left(1-\frac{1}{\nu_{b}(D+1)}\right)=\frac{\nu_{\|}(D-\eta)}{D+1},
$$

where the scaling relation between $\nu_{b}$ and $\eta$ has been used in the second step. Accordingly, we get

$$
H(x) \sim \begin{cases}|x|^{\chi} & x \rightarrow-\infty \\ \text { const } & x \rightarrow 0 \\ x^{\nu_{\|}} & x \rightarrow \infty\end{cases}
$$

The behavior for $x \rightarrow-\infty$ is confirmed by the simulation results in Fig. 6. Note that the applicability of the scaling form (11) to the model without deleterious mutations implies the existence of a second, previously unnoticed scaling regime for adapting spatial populations that appears when $U_{b}$ is large in the sense of $a_{s} U_{d}^{c} U_{b}^{-1 / \varphi} \sim s^{\phi-\alpha} U_{b}^{-1 / \varphi} \ll 1$ [27. The onset of this regime is discernible in the departure of the data from the straight line in Fig. 6.

Summary and conclusions. - In this paper we have studied the speed of adaptation or fitness decline of a population with spatial structure when both beneficial and deleterious mutations are present. We do not take epistasis into account, not only because the absence of epistasis is a prerequisite for the existence of a constant asymptotic speed of fitness change, but also because epistasis provides an alternative and independent mechanism by which Muller's ratchet can be halted [26. Within this setting, we found a general formula Eq. (5) which allowed us to accurately estimate the speed from simulations without invoking any extrapolation.

When both types of mutations are present the speed turns out to take a rather complicated form. In particular, it does not reduce to the sum of the two speeds caused by beneficial or deleterious mutations acting in isolation, as would be the case in small populations where mutations spread and fix independently [44. We developed a scaling theory based on the critical behavior of directed percolation and its unidirectionally coupled multi-species extension. The predicted scaling relations are confirmed numerically for $D=1$ and $D=2$, but the theory should apply in any dimension.

The dramatic enhancement of the efficacy of Muller's ratchet in spatial habitats that we describe is a consequence of the general weakening of natural selection when competition is local rather than global. Experiments aimed at verifying the ratchet mechanism have mostly been conducted in well-mixed populations without spatial structure. The first experiments used RNA viruses, which are distinguished by their large deleterious mutation rates $U_{d} \sim \mathcal{O}(1)$ 45. Since the corresponding selection coefficients are relatively small [46], these systems would be predicted to operate deep in the MR regime.

Deleterious mutation rates in bacteria are much lower. For example, the estimates $U_{d}=5 \cdot 10^{-3}$ and $s=0.03$ were obtained for a mutator strain of Escherichia coli 47. Although our simplified model cannot be expected to be quantitatively applicable to this specific microbial system, it is nevertheless instructive to compare these values to the critical deleterious mutation rate $U_{d}^{c}$ obtained from our simulations. This comparison would place the E. coli strain in the MR regime for 1D populations and in the $\mathrm{HR}$ regime for $2 \mathrm{D}$ populations. Moreover, using Eq. (12) we find that a beneficial mutation rate of $U_{b} \approx 1.2 \cdot 10^{-3} \approx 0.25 \cdot U_{d}$ would be required to reverse the ratchet in one-dimensional populations. Whereas twoand three-dimensional habitats are naturally realized in bacterial colonies, effectively one-dimensional geometries appear, e.g., at the edge of expanding microbial colonies [18, 19, 48, 49.

We are therefore confident that an experimental test of our predictions is principally feasible. In fact, two recent experiments have provided direct evidence for the enhanced effect of deleterious mutations in spatial habitats [48,49, and it has been suggested that the exceptionally low mutation rates in bacteria may have evolved as a consequence of biofilm formation, which requires a more stringent control of deleterious mutations 50. Taken together, these results indicate an important role for deleterious mutations in spatial habitats which should be explored further in experimental and theoretical work.

$$
* * *
$$

S-CP acknowledges the support by the Basic Science Research Program through the National Research Foun- 
dation of Korea (NRF) funded by the Ministry of Science and ICT (Grant No. 2017R1D1A1B03034878) and by the Catholic University of Korea, research fund 2018. JK was supported by DFG within SPP 1590 and CRC 1310. We furthermore thank the Regional Computing Center of the University of Cologne (RRZK) for providing computing time on the DFG-funded High Performance Computing (HPC) system CHEOPS.

\section{REFERENCES}

[1] Muller H. J., Mutat. Res., 1 (1964) 2.

[2] Felsenstein J., Genetics, 78 (1974) 737.

[3] Haigh J., Theor. Popul. Biol., 14 (1978) 251.

[4] Stephan W., Chao L. and Smale J. G., Genet. Res., 61 (1993) 225.

[5] Gordo I. and Charlesworth B., Genetics, 154 (2000) 1379.

[6] Gordo I. and Charlesworth B., Genetics, 156 (2000) 2137.

[7] Rouzine I. M., Brunet E. and Wilke C. O., Theor. Popul. Biol., 73 (2008) 24.

[8] Etheridge A., Pfaffelhuber P. and Wakolbinger A., How often does the ratchet click? Facts, heuristics, asymptotics in Trends in Stochastic Analysis, edited by Blath J., Mörter P. and Scheutzow M., (CUP, Cambridge) 2009

[9] Waxman D. and Loewe L., J. Theor. Biol., 264 (2010) 1120.

[10] Neher R. A. and Shraiman B. I., Genetics, 191 (2012) 1283.

[11] Metzger J. J. and Eule S., PLoS Comp. Biol., 9 (2013) e1003303.

[12] Yu F., Etheridge A. and Cuthbertson C., Ann. Appl. Probab., 20 (2010) 978.

[13] Komarova N. L., Bull. Math. Biol., 68 (2006) 1573.

[14] Gordo I. and Campos P. R. A., Genetica, 127 (2006) 217.

[15] Korolev K. S., Avlund M., HallatscheK O. and Nelson D. R., Rev. Mod. Phys., 82 (2010) 1691.

[16] Otwinowski J. and Boettcher S., Phys. Rev. E, 84 (2011) 011925.

[17] Martens E. A. and Hallatschek O., Genetics, 189 (2011) 1045.

[18] Lavrentovich M. O., Korolev K. S. and Nelson D. R., Phys. Rev. E, 87 (2013) 012103.

[19] Lavrentovich M. O., J. Stat. Mech.:Theory Exp., 2015 (2015) P05027.

[20] Durrett R. and Moseley S., Ann. Appl. Probab., 25 (2015) 104

[21] Durrett R., Foo J. and Leder K., J. Math. Biol., 72 (2016) 1369.

[22] Park S.-C., Simon D. and Krug J., J. Stat. Phys., 138 (2010) 381.

[23] Otwinowski J. and Krug J., Phys. Biol., 11 (2014) 056003.

[24] Wright S., Genetics, 16 (1931) 97.

[25] Fisher R. A., The Genetical Theory of Natural Selection (Clarendon Press, Oxford) 1930.

[26] Jain K., Genetics, 179 (2008) 2125.

[27] PARK S.-C. and Krug J., in preparation, (2018) .
[28] Park S.-C. and Krug J., Proc. Nat. Acad. Sci. USA, 104 (2007) 18135.

[29] Guess H. A., Ann. Prob., 2 (1974) 14.

[30] Park S.-C. and Krug J., Genetics, 195 (2013) 941.

[31] Henkel M., Hinrichsen H. and Lübeck S., Nonequilibrium Phase Transitions. Volume I: Absorbing Phase Transitions (Springer, Berlin) 2008.

[32] Domany E. and Kinzel W., Phys. Rev. Lett., 53 (1984) 311.

[33] Essam J. W., J. Phys. A: Math. Gen., 22 (1989) 4927.

[34] Dickman R. and Tretyakov A. Y., Phys. Rev. E, 52 (1995) 3218.

[35] Alon U., Evans M. R., Hinrichisen H. and Mukamel D., Phys. Rev. Lett., 76 (1996) 2746.

[36] Alon U., Evans M. R., Hinrichsen H. and Mukamel D., Phys. Rev. E, 57 (1998) 4997.

[37] Täuber U. C., Howard M. J. and Hinrichsen H., Phys. Rev. Lett., 80 (1998) 2165.

[38] Goldschmidt Y. Y., Hinrichsen H., Howard M. and Täuber U. C., Phys. Rev. E, 59 (1999) 6381.

[39] Janssen H.-K., J. Phys.: Condens. Matter, 17 (2005) S1973.

[40] LüBeck S., J. Stat. Mech.:Theory Exp., 2006 (2006) P09009.

[41] Jensen I., J. Phys. A: Math. Gen., 32 (1999) 5233.

[42] Wang J., Zhou Z., Liu Q., Garoni T. M. and Deng Y., Phys. Rev. E, 88 (2013) 042102.

[43] Hinrichsen H., Phys. Rev. E, 67 (2003) 016110.

[44] Klatt P., Interference of deleterious and beneficial mutations in spatial habitats (Master thesis, University of Cologne) 2017.

[45] Chao L., Nature, 348 (1990) 454.

[46] Elena S. F. and Moya A., J. Evol. Biol., 12 (1999) 1078.

[47] Trindade S., Perfeito L. and Gordo I., Phil. Trans. R. Soc. B, 365 (2010) 1177.

[48] Lavrentovich M. O., Wahl M. E., Nelson D. R. and Murray A. W., Biophys. J., 110 (2016) 2800.

[49] Bosshard L., Dupanloup I., Tenaillon O., Bruggmann R., Ackermann M., Peischl S. and Excoffier L., Genetics, 207 (2017) 669.

[50] Gralka M., Fusco D. and Hallatschek O., Biophys. J., 111 (2016) 271. 